P5 $\quad$ ACUTE $\beta$-ADRENERGIC STIMULATION OR PERFUSION WITH A CAMP analogue results in immediate and sustained inhibition of mitochondrial permeability transition pore opening

I Khaliulin, MJ Lewis, AP Halestrap, MS Suleiman. School of Clinical Sciences, University of Bristol, Upper Maudlin Street, Bristol, UK

\subsection{6/heartjnl-2018-BSCR.10}

Background Ischaemia/reperfusion ( $/ / R$ ) injury is mediated by opening of the mitochondrial permeability transition pore (MPTP). Experimental studies have shown that a variety of interventions, including ischaemic preconditioning (IP), protect the heart by inhibiting MPTP during reperfusion. Interestingly, we and others have shown that IP has no inhibitory effect on MPTP prior to ischaemia. We have recently shown that acute and transient perfusion of hearts with cell-permeable cAMP analogue confers marked protection against I/R. However, whether this treatment affects MPTP opening is not presently known. The aim of this work was to address this issue.

Methods Isolated Langendorff-perfused rat hearts were perfused with either $0.2 \mu \mathrm{M}$ of the $\beta$-adrenergic receptor agonist isoprenaline (to increase endogenous level of cAMP) for $3 \mathrm{~min}$, or with $7.5 \mu \mathrm{M}$ of the cell-permeable cAMP analogue 8-Br-cAMP-AM (8-Br) for $5 \mathrm{~min}$. Mitochondria were isolated immediately after the treatment. Additional hearts were treated with either intervention and exposed to global ischaemia followed by reperfusion. Mitochondria were isolated from these hearts after $5 \mathrm{~min}$ of reperfusion. MPTP opening was assessed using $\mathrm{Ca} 2+$-induced mitochondria swelling assay or by evaluation of $\mathrm{Ca} 2$ + retention by mitochondria.

Results Both Iso and $8-\mathrm{Br}$ inhibited MPTP opening immediately after the treatment. This inhibitory effect of MPTP opening was also observed at the beginning of reperfusion.

Conclusion In contrast to IP, acute elevation of cAMP level in myocardium either by Iso or $8-\mathrm{Br}$ is associated with desensitisation of MPTP to $\mathrm{Ca} 2+$ retention this effect is maintained during $\mathrm{I} / \mathrm{R}$.

\section{P6 THE ROLE OF PLASMA MEMBRANE CALCIUM ATPASE 1 IN ANGIOGENESIS}

AM Njegic, EJ Cartwright. Division of Cardiovascular Sciences, University of Manchester, Manchester, UK

\subsection{6/heartjnl-2018-BSCR.11}

Angiogenesis, the process of forming new blood vessels, is an important process in both cardiovascular health and disease. A recent student has identified a novel role for plasma membrane calcium ATPase (PMCA) 4 in angiogenesis through mediating endothelial cell migration and tubule formation. However, the role of PMCA1 has yet to be investigated. Here, we aim to establish the role of PMCA1 in angiogenesis and endothelial cell behaviours. We hypothesise that PMCA1 modulates key endothelial cell processes which are associated with the development of new blood vessels.

Knockdown of PMCA1 was achieved in human umbilical vein endothelial cells (HUVECs) using siRNA (si-PMCA1) and confirmed with qPCR. Following PMCA1 knockdown, HUVEC viability and migration was assessed using the MTT assay and wound healing assay respectively. Additionally, the rate of HUVEC proliferation was evaluated by Ki-67 immunofluorescence staining. Finally, apoptosis of HUVECs was investigated using a caspase-Glo3/7 assay.

Transient knockdown of PMCA1 in HUVECs resulted in an $85 \%$ reduction in PMCA1 gene expression. Phenotypically, si-PMCA1 HUVECs display decreased HUVEC cell viability but also reduced apoptosis. Staining for Ki-67 revealed that siPMCA1 HUVECs had a larger percentage of cells active in the cell cycle. Furthermore, loss of PMCA1 impairs migration of HUVECs into the 'wound', 24 hours after the scratch assay was performed.

Overall it appears loss of PMCA1 is detrimental for HUVEC viability and migration which may result in a reduction in angiogenesis, although further work is required to establish the pumps direct role in vessel formation.

\section{P7 PROFILE OF CIRCADIANLY REGULATED METABOLIC GENES IN DYSTROPHIC HEART}

CA Betts, TLE van Westering, M Bowerman, G McClorey, K Meijboom, MJA Wood. Department of Physiology, Anatomy and Genetics, University of Oxford, South Parks Road, Oxford, UK

\subsection{6/heartjnl-2018-BSCR.12}

Duchenne muscular dystrophy (DMD) is a monogenic disorder caused by the lack of the integral structural protein, dystrophin, which results in severe muscle wasting and cardiomyopathy in affected boys. Indeed, cardiorespiratory complications are the predominant cause for mortality in DMD patients. We have recently shown that circadian rhythm is disrupted in dystrophic mice as a direct result of the lack of dystrophin protein. It is well reported that disruption of circadian rhythmicity leads to perturbed metabolism and an array of disorders including obesity, diabetes and cardiovascular disease. Disturbed cardiac metabolism in DMD patients and dystrophic mice is also well described, and thus it would be interesting to learn whether pertinent metabolic genes which are known to be circadianly regulated, are disrupted in dystrophic mice. Here we show for the first time, significant changes in the differential expression patterns of multiple genes involved in free fatty acid and glucose metabolism, in 2 mouse models of DMD compared to control mice. These findings provide the foundation for further research to better understand the metabolic/circadian milieu and its effect on dystrophic heart, so that we may devise strategies to augment cardiac metabolism, in an effort to halt the deterioration in cardiac phenotype.

\section{P8 PROPIONATE ANIONS ACCUMULATED IN PROPIONIC ACIDAEMIA INFLUENCES THE CARDIAC GENE EXPRESSION LANDSCAPE}

KC Park, G Roseman, Y Chung, KL Ford, A Hulikova, P Swietach. Department of Physiology, Anatomy and Genetics, University of Oxford

\subsection{6/heartjnl-2018-BSCR.13}

Propionic acidaemia (PA) is an autosomal recessive disorder characterised by malfunctional mitochondrial propionyl-CoA carboxylase. Consequences of this inborn error of metabolism (IEM) is defective catabolism of propiogenic substrates (branched-chain amino acids, odd-number fatty acids, etc.) leading to mitochondrial accumulation of propionyl CoA and 
its derivatives. Accumulation of propionate derivatives in the cytoplasm and plasma leads to intra- and extracellular metabolic acidosis. The clinical manifestation is severe; arrhythmia and cardiomyopathy (hypertrophic and dilated) are cited as major causes of death. Little is known of the underlying mechanisms. Since butyrate is in the same homologous series as propionate, and is itself a well-established inhibitor of histone deacetylases (HDACs), it is plausible pro-hypertrophic signalling in PA is epigenetically regulated. Therefore, we investigated the effects of propionate on cardiomyocytes.

In preliminary work, culturing neonatal rat ventricular myocytes (NRVMs) in a chemical milieu mimicking PA (6 mM propionate; $200 \mathrm{M}$ ammonium; $18 \mathrm{mM}$ bicarbonate; $\mathrm{pH}$ 7.15) increased significantly cardiomyocyte area. Treating adult rat VMs with PA-medium increased significantly histone-3 lysine-9 acetylation, phenocopying the effects of butyrate. To determine the gene expression profile associated with PA, RNA-sequencing was performed on polyadenylated RNA extracted from NRVMs, treated with control-, butyrate-, or PA-medium. In PA-medium, Differentially Expressed Gene analysis demonstrated $3.0 \%$ of genes were upregulated and $3.0 \%$ downregulated (FDR $<0.0001)$. Gene Ontology Enrichment Analysis using DAVID revealed 'cardiac muscle contraction' and 'metabolic pathways' as being significantly enriched. $1.9 \%$ of genes were upregulated in both PA and butyrate, representing candidates that could be regulated by HDACs. We are currently validating these hits.

\section{P9 HYPERPOLARISED KETONE BODY METABOLISM IN THE RAT HEART}

1,2JJ Miller, ${ }^{1,3}$ YB Ding, ${ }^{1} \mathrm{D}$ Ball, ${ }^{1,4} \mathrm{AZ}$ Lau, ${ }^{1} \mathrm{DJ}$ Tyler. ${ }^{1}$ Department of physiology, Anatomy and Genetics, Sherrington Building, University of Oxford, Oxford; ${ }^{2}$ Department of Physics, Clarendon Laboratory, University of Oxford, Oxford; ${ }^{3}$ Department of Chemistry, Dyson Perrins, South Parks Road, Oxford; ${ }^{4}$ Health Sciences, Sunnybrook Research Institute, Toronto, Canada

\subsection{6/heartjnl-2018-BSCR.14}

Hyperpolarised Magnetic Resonance Spectroscopy (MRS) permits the real time determination of metabolic fluxes in the living heart. In contrast to conventional thermal-equilibrium MRS, the hyperpolarisation technique increases the signal-tonoise ratio of acquired spectra by many orders of magnitude, and therefore allows isotopically labelled probes to be injected into an organism and followed through their subsequent biochemical pathways.

We show here that $[1-13 C]$ acetoacetate and $[1-13 C] \beta$ hydroxybutyrate can be hyperpolarised and probe ketone body metabolism in both the ex vivo perfused and in vivo rat heart. Downstream metabolites were observed within the perfused heart, including acetylcarnitine, citrate, and glutamate. In the in vivo heart, a statistically significant increase in acetylcarnitine production from acetoacetate was observed in the fed state, as well as a potential reduction in glutamate, when compared to fasted controls.

The metabolism of acetoacetate and $\beta$-hydroxybutyrate is known to be altered in various disease states, including diabetic cardiomyopathy, and this proof-of-principle study shows that hyperpolarisation can probe the role of ketone bodies in the diseased heart. The increased rate of acetylcarnitine production following feeding is consistent with its reported role as a store of acetyl moieties should they be abundant in a post-prandial state, into which ketone oxidation is directed. In the fasted state, apparent glutamate levels were higher, which is consistent with an increased flux of ketone bodies into the TCA cycle during fasting.

Further work will aim to quantify these fluxes, and explore the role of ketone bodies in animal models of cardiac disease, such as diabetic cardiomyopathy.

\section{P10 MIR-133A OVEREXPRESSION IMPAIRS ENDOTHELIAL CELL MIGRATION AND TUBE FORMATION IN VITRO}

${ }^{1}$ Sathishkumar Kurusamy, ${ }^{1}$ Jude C Ihugba, ${ }^{1}$ Angel L Armesilla*, ${ }^{2}$ James M Cotton*.
${ }^{1}$ Cardiovascular Molecular Pharmacology Laboratory, School of Pharmacy, Research Institute
in Healthcare Science, Faculty of Science and Engineering, University of Wolverhampton,
Wolverhampton, UK; ${ }^{2}$ Department of Cardiology, Heart and Lung Centre, New Cross
Hospital, Wolverhampton, UK; 'Equal senior authors

10.1136/heartjnl-2018-BSCR.15

Emerging evidence indicates that short ( 22 nucleotides) noncoding RNA molecules called microRNAs (miRNAs) play a key role in the regulation of post-ischaemic angiogenesis. miRNAs control gene expression by binding to complementary sequences of specific mRNA transcripts, leading to degradation or translational repression of the targeted mRNA. miR-133a is highly expressed and plays a crucial role in skeletal and cardiac muscle biology. The expression level of miR-133a in vascular endothelial cells is very low under physiological conditions. However, multiple cardiovascular risk factors including oxidized-LDL, pro-inflammatory cytokines, hyperglycemia, dyslipidemia, and hyperhomocysteinemia induce aberrant miR-133a expression in endothelial cells leading to endothelial dysfunction. Endothelial dysfunction in cardiovascular ischaemic disease is often accompanied by impairment of reparative angiogenic processes.

Here, we have evaluated the role of aberrant miR133a expression in VEGF-mediated angiogenesis. We show that exogenous expression of miR-133a in Human Umbilical Vein Endothelial Cells (HUVEC) significantly reduces VEGF-induced endothelial cell proliferation. Moreover, adenoviral-mediated delivery of cardiac miR133a into endothelial cells inhibits HUVEC cell motility and tubular morphogenesis. Interestingly, downregulation of phosphorylation (activation) of the signalling protein Erk1/2 by miR-133a was detected in VEGF stimulated HUVEC cells.

Our results demonstrate that aberrant expression of miR133a has an anti-angiogenic effect in endothelial cells, and strongly suggest that targeted strategies to suppress ectopic expression of miR-133a in the dysfunctional endothelium might have important therapeutic applications to improve reparative angiogenic process in patients suffering from ischaemic cardiovascular disease.

Acknowledgement This work was supported by the Rotha Abraham Bequest Charity 